\title{
PREVALENCIA DE MICOSIS ORAL POR Candida albicans, EN PACIENTES BAJO TRATAMIENTO EN EL HOSPITAL JOSÉ CARRASCO ARTEAGA (IESS)
}

\section{PREVALENCE OF ORAL MYCOSIS BY Candida albicans, IN PATIENTS UNDER TREATMENT AT JOSÉ CARRASCO ARTEAGA HOSPITAL (IESS)}

\author{
Mora-Naula William $^{1 *}$, Reinoso-Quezada Santiago ${ }^{2}$ \\ ${ }^{1}$ Odontólogo egresado de la Universidad Católica de Cuenca. Ecuador \\ ${ }^{2}$ CD. Esp Ciurgía Máxilo Facial- Docente en la Universidad Católica de Cuenca. Ecuador \\ *willyfab@hotmail.com
}

\begin{abstract}
Resumen
OBJETIVO: Determinar la prevalencia de micosis oral por Candida albicans en pacientes entre 25 - 65 años de edad con cáncer que se encuentran bajo radioterapia (RT) y/o quimioterapia (QT), en el hospital José Carrasco Arteaga en el año 2015. MATERIALES Y MÉTODOS: El presente estudio es de tipo descriptivo de corte transversal. La muestra cuenta con una cifra de 98 historias clínicas de pacientes atendidos en el Hospital José Carrasco Arteaga desde enero 2014 a junio 2015. RESULTADOS: Desde Enero 2014 a Junio 2015, 81 historias clínicas de pacientes que recibieron RT y/o QT, manifestando Candida albicans, determinando una prevalencia de $82.7 \%$ de la muestra; el grupo de sexo masculino registró a 48 integrantes representando el $59.25 \%$, el grupo femenino reportó 33 integrantes reportando el $40.75 \%$; los pacientes integrantes al grupo "mayores a 60 años de edad" registraron 46 personas, ocupando el $47 \%$ de la muestra. Un grupo de 66 personas recibieron RT manifestaron infección por Candida albicans. Pacientes sometidos a radiaciones comprendidas entre 51-70 Gy fueron 28 individous, representando el $41.5 \%$ de la muestra estableciéndolo como el grupo con mayor relación. Un grupo 48 personas recibieron QT manifestaron infeción por Candida albicans; donde destaca el uso del carboplatino en 15 personas representando $31.3 \%$ de la muestra, estableciendo una relación directa del fármaco con la enfermedad fúngica. La ubicación frecuente de Candida albicans en mucosa oral se registró en 57 casos, determinando una prevalencia de $44.9 \%$ de la muestra. CONCLUSIONES: La Candida albicans esta preste en el $82.7 \%$ de los pacientes bajo tratamiento contra el cáncer, afectando mayormente al sexo masculino. Presenta con más frecuencia en pacientes mayores a 60 años de edad, guarda mayor relación con tratamientos de radiación en dosis entre 51-70 Gy.
\end{abstract}

Palabras clave: Prevalencia, frecuencia, micosis, Candida albicans, radioterapia, quimioterapia, cáncer.

\begin{abstract}
AIM Determine the prevalence of oral mycosis by Candida albicans (CA) in patients between 25-65 years of age with cancer who had radiation therapy (RT) \& chemotherapy $(C T)$ in José Carrasco Arteaga hospital 2015. MATERIALS AND METHODS: In this paper we use a descriptive cross-sectional study. The sample exhibition includes 98 medical records of patients treated at the José Carrasco Arteaga Hospital from January 2014 to June 2015. RESULTS: From January 2014 to June 2015, 81 medical records of patients who received $R T \& Q T$, developed Candida albicans infection, determining a prevalence of $82.7 \%$; male group recorded 48 members representing $59.25 \%$, the female group recorded 33 members, reporting their prevalence by $40.75 \%$; patients to the group members "above 60 years old" recorded 46 people, occupying $47 \%$ of the sample. A group of 66 people received RT developed Candida albicans infection. Patients undergoing radiation between 51-70 Gy, 28 individuals were affected, representing 41.5\% of the sample establishing as the group with the highest record. A group of 48 people received QT developed Candida albicans infection; in this group 15 cases were treated with carboplatin representing $31.3 \%$ of the sample, establishing a direct relationship of the drug with the Candida albicans infection. The frequent location of Candida albicans at the oral mucosa was recorded in 57 cases determining a prevalence of $44.9 \%$ of the sample. CONCLUSIONS: The CA was present on $82.7 \%$ patients treated with RT and CT. It manifests more on males than females. Occurs more frequently in patients over 60 years of age, related to radiation treatments at doses between 51-70 Gy.
\end{abstract}

Key words: Prevalence, frequency, fungal infections, Candida albicans, radiotherapy, chemotherapy, cancer. 


\section{INTRODUCCIÓN}

La radioterapia y la quimioterapia son de uso cotidiano en el tratamiento contra el cáncer, estos a su vez presentan una serie de efectos secundarios como la micosis por Candida albicans, que sus manifestaciones pueden causar alteraciones en las funciones como la masticación, deglución, fonación y halitosis perjudicando de manera indirecta la relación social; en la ciudad de Cuenca no se ha encontrado un reporte con una cifra que determine la cantidad de pacientes que manifiestan Candida albicans como efecto de la RT y/o QT; pese al conocimiento de la existencia de esta. La candidiasis es una enfermedad oportunista, muy frecuente en nuestros días, dependiente de factores ajenos a los microorganismos; ${ }^{1}$ estos se presentan como efecto secundario a la RT y/o QT, ya que el efecto emitido por la máquina de radiación o por el uso del químico no agrede únicamente a las células cancerígenas, sino que también se ven afectadas las células alrededor de la zona afectada por el cáncer; en pacientes que presentan cáncer de cabeza y cuello (C.C.C), la radiación y los químicos administrados agreden a las células de las glándulas salivales provocando la disminución en la producción de saliva o la falta completa de esta (xerostomía) dando paso a la neutropenia, factores que permiten crear un habitad favorable para la Candida albicans. En determinadas circunstancias, se producen cambios bioquímicos en la mucosa oral, que favorece el crecimiento de estos gérmenes"; ${ }^{2}$ como son el desequilibrio del pH salival de 7 a 6.5 , permitiendo el establecimiento del biofilm fúngico a través de sus mecanismos de adhesión molecular (CR3), para luego permitir su crecimiento y desarrollo. Una vez establecida Sapp Philips las describe como "Placas cremosas blanquecinas, de epitelio descamativo sobre mucosa eritematosa. ${ }^{3}$ La RT es un método de tratamiento que consiste en el empleo de radiaciones ionizantes, para destruir las células cancerosas o disminuir el tamaño de los tumores, este actúa agrediendo el material genético de las células en el área, imposibilitando su crecimiento y división. ${ }^{4}$ La QT dentro de la Oncología está dirigida a impedir que las células neoplásicas se reproduzcan, para lo cual provocan una alteración en la síntesis proteica, ácidos nucleicos o en su misma división, pero también afecta a células no cancerosas, dentro de las cuales se presentan las células de defensa con los linfocitos T,C,D etc. ${ }^{5}$ Alterando el sistema de defensa en el sitio, además de otros tipos de células como las ubicadas en las glándulas salivales, de ligamento periodontal papilas gustativas, folículos pilosos, etc.

\section{MATERIALES Y MÉTODOS}

Para determinar la existencia de Candida se realiza exámenes de microscopía con Hidróxido de potasio al $20 \%$ (KOH $20 \%$ ), el cual es tomado con hisopo en él sitio de la lesión activa en cavidad oral y enjuagado con solución salina para Candida; transportado en contenedor estéril, se cultiva por 24 horas a temperatura ambiente siendo este el medio selectivo para levaduras; luego de analizar el cultivo macroscópicamente, se procede al análisis con $\mathrm{KOH}$ al $20 \%$ en microscopio convencional diafragmado el condensador para aumentar el contraste. El procedimiento ejecutado fue, mezclar $\mathrm{KOH} 10 \mathrm{~g}$ en $40 \mathrm{ml}$ de agua destilada, añadiendo $20 \mathrm{ml}$ de Glicerol y aumentar agua destilada hasta $100 \mathrm{ml}$. Se coloca la muestra fúngica en el porta, añadir una gota de la mezcla de $\mathrm{KOH}$ poner un cubre y calentarlo a 55 grados centigrados por 10 minutos, observar a través de microscopio a bajo aumento (10x), confirmado a 40x. ${ }^{6}$ Para la identificación de género y especie, Se utilizó el medio cromogénico CHROMagar Candida, tiñéndose de color verde la especie albicans, dando como resultado positivo. ${ }^{7}$

\subsection{POBLACIÓN}

Pacientes con cáncer, que reciben tratamiento con radiación y quimioterapia en el hospital JOSE CARRASCO ARTEAGA (IESS) 98 casos, durante el periodo enero 2014 Junio 2015.

\subsection{MUESTRA}

81 pacientes diagnosticados con MICOSIS ORAL POR C. albicans, como efecto secundario al tratamiento de radioterapia o quimioterapia durante el periodo enero 2014 - junio 2015, por lo tanto la muestra es discrecional.

\subsection{OBJETIVO GENERAL}

Establecer la prevalencia de Candida albicans en pacientes bajo RT y/o QT tratados en el Hospital José Carrasco Arteaga durante el periodo enero 2014 - junio 2015.

\subsection{OBJETIVOS ESPECÍFICOS}

1) Crear una base de datos con las historias clínicas de pacientes que manifiestan Candida albicans como efecto a la RT y/o QT.

2) Clasificar de acuerdo a edad y sexo donde se manifiesta mayormente la Candida albicans.

3) Establecer la dosis de radiación y el fármaco con mayor relación a la Candida albicans.

4) Localizar la estructura con más frecuencia de manifestación de Cándida albicans.

\subsection{CRITERIOS DE INCLUSIÓN}

Todos los pacientes con Cáncer de Cabeza y Cuello (C.C.C) comprendidos entre 25 y 65 años de edad, que reciben Radioterapia o Quimioterapia en el Hospital José Carrasco Arteaga y manifiestan micosis por Cándida albicans.

\subsection{CRITERIOS DE EXCLUSIÓN}

Pacientes que presenten osteoradionecrosis, mucositis, dolor oral por tratamiento contra el cáncer, las infecciones víricas: virus herpes simple (VHS), virus varicelazóster (VVZ), virus de Epstein-Barr (VEB), citomegalovirus (CMV), pacientes menores de 25 años. 


\begin{tabular}{|c|c|c|}
\hline Válidos & Frecuencia & Porcentaje válido \\
\hline Varón & 55 & 56,1 \\
\hline mujer & 43 & 43,9 \\
\hline Total & 98 & 100 \\
\hline
\end{tabular}

Tabla 1. Porcentaje de varones y mujeres de la población.

\begin{tabular}{lcccc}
\hline & \multicolumn{2}{l}{$\begin{array}{c}\text { Presencia de C. } \\
\text { Albicans }\end{array}$} & Total & Porcentaje \\
Sexo & No & $\mathrm{Si}$ & & \\
\hline Varón & 7 & 48 & 55 & $59.25 \%$ \\
Mujer & 10 & 33 & 43 & $40.75 \%$ \\
Total & 17 & 81 & 98 & $100 \%$ \\
\hline
\end{tabular}

Tabla 2. Porcentaje de varones y mujeres de la muestra que presentan o no C. albicans

\section{RESULTADOS}

La población analizada estuvo conformada por las historias clínicas de 98 pacientes tratados en el área de oncología con RT y QT; los resultados se analizaron en el programa SPSS 21, además de realizar la revisión de las historias clínicas digitales en el sistema interno del Hospital José Carrasco Arteaga AS 400; y sus resultados son:

Varones, $59.25 \%$ con presencia de Candida albicans bajo tratamiento contra el cáncer y mujeres $40.75 \%$.(tabla 1 )

41 - 65, años grupo etario con mayor presencia de Candida albicans bajo tratamiento contra el cáncer.(tabla 2,3)

Radiación con 51 - 70 Gy, dosis con mayor relación con Candida albicans bajo tratamiento contra el cáncer.(tabla 4 y gráfico 2)

Carboplatino, fármaco con mayor relación con Candida albi-

\begin{tabular}{lll}
\hline \multicolumn{2}{c}{ Clasificación por edades de pacientes diagnosticados con C. Albicans bajo } \\
tratamiento contra el cáncer
\end{tabular}

Tabla 3. Sectores por grupo de edades.

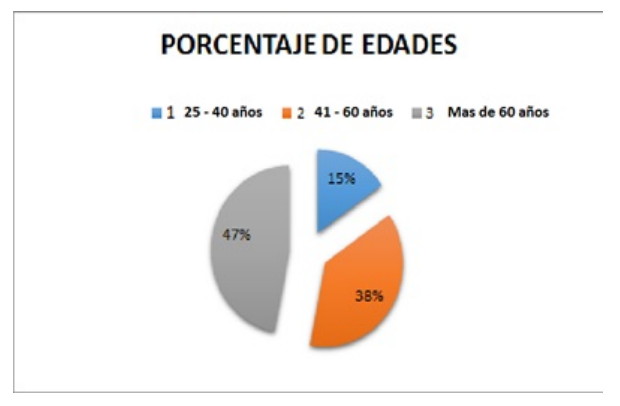

Grf. 1. Representación del porcentaje por edades.

\begin{tabular}{ccc}
\hline DOSIS & Frecuencia & Porcentaje \\
\hline $\mathbf{1 0 - 2 0}$ Gy & 6 & 9,2 \\
$\mathbf{2 1}-\mathbf{4 0}$ Gy & 14 & 21,5 \\
$\mathbf{4 1 - 5 0}$ Gy & 15 & 23,1 \\
$\mathbf{5 1}-\mathbf{7 0}$ Gy & 28 & 41,5 \\
más de 70 Gy & 3 & 4,6 \\
Total & 66 & 100,0 \\
\hline
\end{tabular}

Tabla 4. Dosis de radiación usadas.

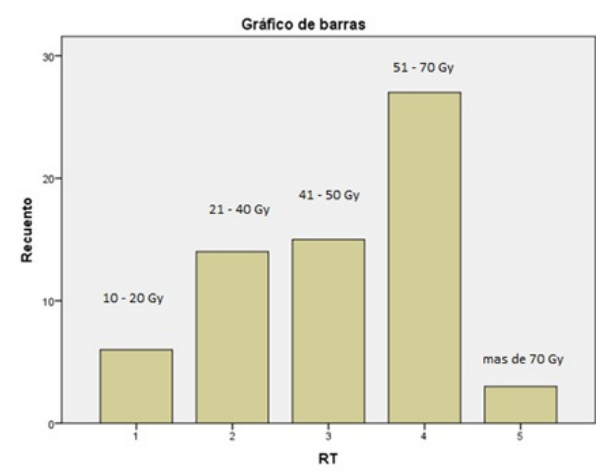

Grf. 2. Representación del porcentaje de dosis de radiación.

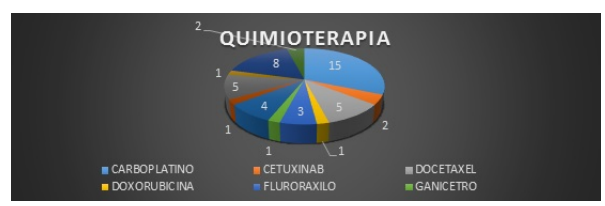

Grf. 3. Representación del porcentaje usado de fármacos en quimioterapia. 


\begin{tabular}{ccc}
\hline Ubicación & Frecuencia & Porcentaje \\
\hline comisuras & 1 & 0,8 \\
labios & 3 & 2,4 \\
lengua & 31 & 24,4 \\
mejillas & 3 & 2,4 \\
mucosa & 57 & 44,9 \\
paladar & 7 & 5,5 \\
piso de boca & 25 & 19,7 \\
Total & 127 & 100 \\
\hline
\end{tabular}

Tabla 5. Ubicación de C. Albicans

cans bajo tratamiento contra el cáncer.(gráfico 3)

Mucosa oral, zona mayor número de manifestaciones de Candida albicans(tabla 5)

Como resultado final se obtuvo que la prevalencia de Candida albicans es de 82.7 en el Hospital José Carrasco Arteaga, en pacientes registrados de enero 2014 a junio 2015.

\section{DISCUSIÓN}

Se considera que los estudios realizados sobre la Candida albicans en el hospital José Carrasco Arteaga en la ciudad de Cuenca Ecuador, constituye un campo novedoso y reciente, debido a la no existencia del mismo previamente, luego de revisar en estudios realizados en otros países latinoamericanos, podemos deducir que los niveles obtenidos acerca de la prevalencia de Candida albicans en este estudio se encuentran superiores. La prevalencia en el Hospital José Carrasco Arteaga es de $82.7 \%$ en el plazo de un año y medio. Rueda Gordillo et al. 2008, estado de Yucatán México; realizó un estudio donde se encontró una prevalencia de $50.3 \%$ de Candida albicans, en pacientes con C.C.C. Los pacientes con cáncer poseen factores que aumentan el riesgo de adquirir infecciones causadas por micro organismos oportunistas pues son pacientes inmunosuprimidos, bajo tratamiento antineoplásico, sometidos a cirugías extensas, con catéteres intravasculares de larga estancia y otros dispositivos que rompen las barreras naturales. ${ }^{2}$ Ynca Cahuana Jessica. 2005, Perú; analizó la frecuencia de candidiasis pseudomembranosa en pacientes con C.C.C los mismos que se encontraban bajo tratamiento de radiación, se obtuvieron 30 muestras en el Servicio de Radioterapia del Instituto Nacional de Enfermedades Neoplásicas y el Hospital Nacional Edgardo Rebagliati
Martins; Hallando presuntivamente las especies de Candida. ${ }^{8}$ Confirmando que la especie de cándida relacionada a candidiasis pseudomembranosa bucal más frecuente, fue Candida albicans $60 \%$; la aparición de candidiasis pseudomembranosa clínica durante el tratamiento de radiación para el cáncer de cabeza y cuello, fue con una dosis mediana de radiación de 3000 cGY. Cortés A Jorge et al. 2003, Colombia. Identificaron diez hongos: Ocho aislamientos de Candida albicans y Candida tropicalis, un aislamiento de Trichosporon beigelii y uno de Aspergillus terreus. Estudio realizado en los pacientes con neutropenia y diagnóstico de cáncer, con 44 aislamientos, representando el 52\%. "Correia Jham Bruno, Brasil; Estudio realizado demuestra que existe un aumento de Candida en pacientes que se encuentran bajo RT contra el C.C.C., indica que en la consulta inicial existe el $43 \%$ de pacientes que lo presenta, pero durante el tratamiento alcanzó una cifra de $62 \%$, y finalmente durante las visitas de control, las manifestaciones de Candida fueron de $75 \%$. Los pacientes del estudio fueron controlados hasta 12 meses después, donde se observó que en los primeros 6 meses existió un aumento de manifestaciones, pero luego, una revisión a los 12 meses las cifras descendieron por debajo de lo normal $30 \%$. $^{10}$

\section{CONCLUSIONES}

Se registraron 81 pacientes que manifestaban Candida albicans relacionada con los tratamientos contra el cáncer, es así que se determina que la prevalencia de micosis oral por Candida albicans en pacientes adultos bajo tratamiento de radioterapia y quimioterapia en el hospital José Carrasco Arteaga (IESS) de la ciudad de Cuenca - Ecuador; enero 2014 - junio de 2015 es de $82.7 \%$. Se creó una base de 
datos con las fichas únicamente de pacientes con cáncer de Cabeza y cuello que han recibido RT o QT en hospital José Carrasco Arteaga, desde enero 2014 a junio 2015 con el fin de lograr relacionar y obtener resultados cuantitativos actuales. Se logró determinar que, la Candida albicans afecta en la mayor parte al género masculino que al femenino, que tratados con RT y/o QT. La micosis por Candida albicans en pacientes adultos bajo RT o QT se manifestó mayormente en el tercio grupal de más de 60 años de edad.

Evidenciamos que los pacientes con C.C.C del hospital José Carrasco Arteaga reciben mayormente la radiación como terapia, superando al uso de la quimioterapia, cabe recalcar que pacientes que son administrados con dosis a partir de 10 GY se tornan susceptibles a la alteración de su sistema de defensa y colonización de la Candida albicans, encontrando mayor frecuencia en dosis de 51 a 70 GY. Ubicando este grupo como los de mayor riesgo. Dentro de la quimioterapia el carboplatino es el fármaco más usado y esta seguido de temozolamida, de acuerdo a los registros dentro del HOSPITAL JOSÉ CARRASCO ARTEAGA. La Candida albicans se manifiesta con mayor frecuencia en la mucosa oral, seguida en lengua y paladar.

\section{RECOMENDACIONES}

Realizar un estudio en años posteriores con el fin de compararlo con el actual, analizando si esta cifra aumenta o disminuye. Lograr determinar la prevalencia total del Ecuador de la Candida albicans como efecto a la RT y/o QT.

Conflicto de intereses y financiamiento Los autores declaran no tener conflicto de intereses, haber cumplido con los requisitos de autoría y haber autofinanciado este artículo.

\section{Referencias}

1 Rueda G F, Hernández S E. Prevalencia de Candida albicans aislada de la cavidad oral de pacientes con cáncer. Revista odontológica latinoamericana 2008. Vol 0, num 2, pag 38 41; citado 12/7/2015; disponible en: Documento disponible

2 Rodríguez J 1, Miranda J 2, Morejón H 3 y Santana J C4, Candidiasis de la mucosa bucal. Rev Cubana Estomatologia. 2002 v.39 n.2 citado 3/7/2015 disponible en: Documento disponible

3 Sapp J P; Eversole L R; Wysocki G P. infecciones micóticas; patologia oral y maxilofacial contemporanea; segunda edicion;Pag 204; citado 09/06/15

4 Oddo A, Zuñiga C, Fernandez E, Garcia R; CANDIDA ALBICANS; blogspost. [internet];2007 citado 10/07/2015; disponible en: Documento disponible

5 Cancer.Net Editorial Board [INTERNET]. Salivary Gland Cancer: Risk Factors. Cancer.Net citado 15/12/2015 disponible en: Documento disponible

6 Cuenca M, Gadea I, Martin E, Pemán J, Poton J, Rodriguez J L; Diagnostico microbiológico de las micosis y estudios de sensibilidad a los antifúngicos. En: Cercenado E, Cantón $\mathrm{R}$, editores. Procedimientos en microbiología Clínica. 21va edición. 2006. p. 15-27.

7 Biasoli M; Candidiasis. En: Martin A, editor. Candidiasis o Candidosis. [INTERNET]; 14/03/2013. p. 20 - 24

8 Ynca Cahuana J; Candidiasis Boca - Enfermedades Cáncer - Tratamiento Radioterapia, Universidad Nacional Mayor de San Marcos 2006. Citado 12/9/2015; disponible en: Documento disponible

9 Cortés A J. 1 , Cuervo I S.1 , Arroyo P 2 , Quevedo R 2; hallazgos microbiológicos en pacientes con neutropenia febril; revista colombiana de cancerología 2003; 7(4):5-11; citado 20/10/2015

10 Correia J B I; da Silva Freire A R, Complicações bucais da radioterapia em cabeça e pescoço, Revista Brasileira de Otorrinolaringologia Print version ISSN 0034-7299. vol.72 no.5 SãoPaulo Sept/Oct. 2006; citado 9/9/2015; disponible en: Documento disponible

1 Böhme A, Karthaus M, Hoelzer D: Antifungal prophylaxis in neutropenic patients with hematologic malignancies. Antibiot Chemother 50: 69-78, 2000. Citado 12/07/2015; disponible en: Documento disponible

12 Mittal BB, Pauloski BR, Haraf DJ, et al.: Swallowing dysfunction-preventative and rehabilitation strategies in patients with head-and-neck cancers treated with surgery, radiotherapy, and chemotherapy: a critical review. Int J Radiat Oncol Biol Phys 57 (5): 1219-30, 2003.

13 IPRO: tecnología avanzada sobre vida y calidad [Internet].Córdova IPRO:2011, [CITADO 11/8/2015 disponible en: Documento disponible

14 ACS: American cancer society [internet]. Radiation treatment; USA actualizado en 2014; [citado 13/8/2015]; disponible en: Documento disponible

15 Lalla RV, Latortue MC, Hong $\mathrm{CH}$, et al.: A systematic review of oral fungal infections in patients receiving cancer therapy. Support Care Cancer; USA 2010. [internet];[CITADO15/8/2015]; disponible en: Documento disponible

16 Mota G A, Sociedad Mexicana de oncologia A.C 2012, [citado 18/08/2015]; disponible en: Documento disponible

17 Castañón Olivares L R. Unidad de Micología Departamento de Microbiología y Parasitología, Facultad de Medicina, UNAM lrcastao@unam.mx 10 agosto 2015

18 ASCO: cáncer.net[internet];junta editorial de cáncer.net; Estados Unidas de America:2005-2014 Amercan Society of Clinical Oncology (ASCO); citado 28 de septiembre de 2015; disponible en: Documento disponible

19 NIH: instituto nacional del cáncer en EEUU[internet]; USA: citado 28 de septiembre de 2015; disponible en: Documento disponible

20 Lazarde LJ: candidiasis multifocal bucal. Acta odontológica venezolana[internet]; 2003 citado [6/10/2015];41(2); disponible en: Documento disponible

21 Mujica M.T*, Finquelievich J.L, Jewtuchowicz V, Iovannitti 
C.A. Prevalencia de Candida albicans y Candida no albicans en diferentes muestras clínicas. Período 1999-2001 Rev. argent. microbiol. v.36 n.3 Ciudad Autónoma de Buenos Aires jul - sep 2004; [citado 28/8/2015] disponible en: Documento disponible

22 Carrión A.* Rey O; Patología oral asociada a la sequedad bucal Avances en Odontoestomatología versión impresa ISSN 0213-1285 Av dontoestomatol vol.30 no.3 Madrid mayo-jun. 2014; [citado 28/8/2015]; Documento disponible

23 Aguado J M; Ruiz C I, Muñoz P, Mensa J, Almirante B, Vázquez L; Guidelines for the treatment of invasive candidiasis and other yeasts; Spanish Society of Infectious Diseases and Clinical Microbiology (SEIMC) [internet]; Actualized en 2010; citado en 24/9/2015;

24 Raymond J. Fonseca; oral and maxilofacial surgery. Volume 5. USA: editorial Robert V. Walker;2010

25 Corpus K1, Hegeman-Dingle R, Bajjoka I. Candida kefyr, an uncommon but emerging fungal pathogen: report of two cases; NCB; pubmed [internet]; 2004 [fecha de consulta 10/10/2015]; Documento disponible

26 Pfaller M. A. , Diekema D. J. , Gibbs D. L. , Newell V. A. , Nagy E. , Dobiasova S;Candida krusei, a MultidrugResistant Opportunistic Fungal Pathogen: Geographic and Temporal Trends from the ARTEMIS DISK Antifungal Surveillance Program, 2001 to 2005; NCBI: journal of clinical microbiology [internet]; 2008 [fecha de consulta $10 / 10 / 2015$

27 Quesada C, Murillo L, Ureña M, Vargas E; CANDIDA DUBLINIENSIS, BINASS; revista médica de costa rica [internet]; 2007 [ fecha de consulta 10/10/2015];Documento disponible

28 Paul L. Fidel, Jr., 1, *Jose A. Vazquez, 2 and Jack D. Sobel2 ; Candida glabrata: Review of Epidemiology, Pathogenesis, and Clinical Disease with Comparison to C. albicans; NCBI: Clinical Microbiology Reviews[internet]; 1999 [fecha de consulta 10/10/2015]; Documento disponible

Recibido: 01 de Julio de 2016

Aceptado: 29 de Julio de 2016 